\title{
Biomarkers: A Comprehensive Review and Its Role in Cancer Treatment
}

\author{
Shashikant Patil \\ Member IEEE, Mumbai, India \\ Chinmaya Vyas \\ SVKM's NMIMS, Mumbai, India \\ Bhumija Singh \\ SVKM's NMIMS, Mumbai, India
}

\begin{abstract}
The paper gives an overview regarding biomarkers their rudimentary characteristics and the abridgment between biomarkers and cancer treatment. The different type of biomarkers and their utility in different environments and conditions gives us cutting edge in terms of cancer treatment. Biomarkers have indeed brought a revolution in the field of medical diagnosis. They have proven to be harbingers of change in the clinical industry. They have a dual functionality and may be used alone or in combination to monitor the health or disease state of an individual. Various clinical assessment techniques have been carried out using biomarkers. The paper gives brief review about biomarkers and also studies various developments that have been done in the recent years.
\end{abstract}

\section{INTRODUCTION}

A Biomarker is a diagnostic that is objectively measured and evaluated as an indicator of normal biological or pathogenic processes and responses to a therapeutic intervention with high accuracy. Biomarkers are the characteristic measures used to perform a clinical assessment and other objectives of person's health status, stratify patients and help streamline therapeutic intervention.

Biomarkers are key cellular events that link a specific environmental exposure to a change in the state of proteins which in turn correlates with the progression of the disease. Biomarkers play an important role in comprehending the relationships between exposure of an individual to environmental chemicals, the development of chronic human diseases, and the homogeneous classification of subgroups for the disease. Much research and progress has been made in identifying and corroborating new biomarkers that can be used in humankind studies of environmental disease. Steven A. Soper mentions that the utility of any bio sensing platform is intimately dependent on the infallibility of biomarker(s) for achieving diagnoses with high accuracy [1]. The outbreak in molecular biology and associated technologies has led to a deeper understanding of human cancer and malignancy and potential biomarkers that can be used for diagnosis. Despite these advances, progress in implementing useful diagnostics in the field of cancer has been slow. For example, the number of markers that has been recommended under the guidelines of American Society of Clinical Oncology for regular clinical use in colorectal and breast canceris significantly short. A comprehensive understanding of these conservative guidelines sheds insight into pragmatic issues and complications for development of cancer diagnostic platforms. Notably, biomarkers must not only mark the presence of a tumor or cancer, but should also determine the stage of tumorigenesis. A novel biomarker might have one or more utilities, each requiring a discrete set of investigations. These utilities include risk assessment, prognosis of early or metastatic disease and screening before diagnosis. During diagnosis it incorporates prediction of benefit from specific therapies, prediction of toxicity related to the therapy and monitoring of clinical course in patients with established metastatic disease. One of the biggest issues in marker implementation is reproducibility of the assay or among different assays, for a given cancer-related change. Intermittently, from one study to the next, there are many assay formats for a particular marker. Within the same format there could be different reagents. For the same reagents there might be different analytical criteria and cut-offs for positive versus negative, 
mentions that Biomarkers (short for biological markers) are biological measures of a biological state. A biomarker must have high specificity, sensitivity, accuracy and robustness to become a clinically approved test. Recent developments in genomics, proteomics, microassay technologies and informatics assure rapid identification of polymorphic variants or alterations in genes expression influencing both response and susceptibility to carcinogens [60].

\section{BIOMARKERS}

The advancement in the biomarkers has led to its application in every medical arena. Every biological system (like the cardiovascular system, metabolic system, nervous system, genetic disorder or the immune system) has its own explicit biomarkers. Many of these biomarkers are quite easy to measure and become part of regular medical examinations.

For example, a general health check-up comprise of assessment of blood pressure, heart rate, cholesterol, triglycerides and fasting glucose levels. Body measurements such as weight, BMI, and waist-to-hip ratio are consistently used for evaluating conditions such as obesity and metabolic disorders.

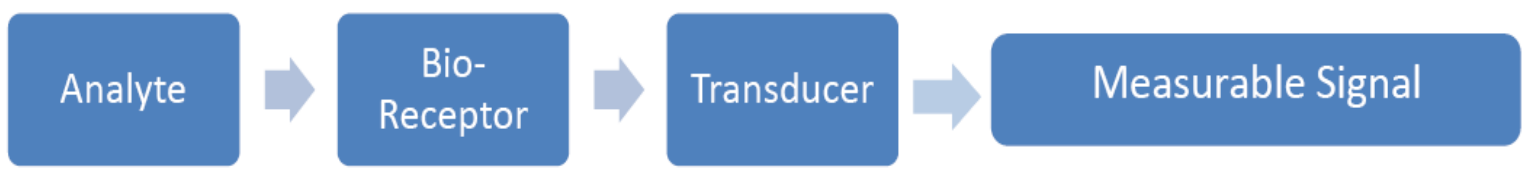

\subsection{Biomarkers as Health and Disease Predictors}

Biomarkers are used to foretell serious illnesses such as diabetes and cardiovascular disease. Each diagnostic biomarker indicates whether there is a disease or a change in health state and can be combined to provide a detailed picture of how healthy a person is and the susceptibility of the disease.

\subsection{Biomarkers in cancer detection and drug development}

The principles of biomarkers have been applied to every stage of the disease starting from the detection, screening, and diagnosis till the treatment and monitoring of cancer. Conventionally, anticancer drugs were agents that killed both cancer cells and healthy cells. However, more specific and targeted therapies have now been developed which are directed to kill cancer cells only, sparing healthy cells. The comprehensive assessment of a typical biomarker in cancer helps in the development of therapies that can make the biomarker drug specific. This minimizes the risk of toxicity and reduces the cost of treatment. In cancer research, genetic studies are important because genetic abnormalities so often determine the development of cancer. Certain DNA or RNA markers are helpful in the detection and treatment of specific cancers.

\subsection{Characteristics of an 'ideal' biomarker}

An important consideration in the use of Biomarker is the practicality of subsequent analysis, which is likely to be far more successful and robust if based on a physiological or biochemical essay that can be carried out with minimal training and perexitance amusement. Biomarkers must be robust biologically and technically to transfer across patient group and across international boundaries. A biomarker needs to be accessible and ideally could be non-invasive. The ideal biomarker for cancer must be capable of determining predispositions, early detestations, assessment of prognosis, and drug response. It would be more advantageous if the biomarkers serve a target for drug development. Desirable characteristics of molecular markers for cancer are shown in table below. No biomarker meets all these requirements but these should be kept in mind for selection of biomarkers to perform the diagnostic tests. There is an urgent need for cancer bio marks with more specific and sensitive diagnostic capability particularly for early stage cancer.

\subsection{Desirable characteristics of biomarkers in cancer}

\section{- Specific}

- High myocardium/serum ratio

- Not present in non-cardiac tissue, even pathologically

- Differentiation of cardiac toxicity (acute versus chronic, necrosis, hypertrophy, rhythm) 
- Sensitive

- Zero baseline

- Marker of 'early,' reversible cardio toxicity

- Immediate release with injury

- Predictive

- Long half-life in blood

- Gives indication of reversibility

- Release proportionate to extent of injury

- Robust

- Rapid, simple, accurate and inexpensive detection in all relevant species

Table1. Comparative sensitivity and specificity data of various widely used biomarkers [58]

\begin{tabular}{|c|c|c|c|c|}
\hline Test & Sensitivity ${ }^{*}$ & Specificity ${ }^{*}$ & $\begin{array}{l}\text { Therapeutic, prognostic \& diagnostic use } \\
\text { of biomarker }\end{array}$ & Ref. \\
\hline CYP2C9*1/*2/3 & $46 \%$ & $69 \%$ & $\begin{array}{l}\text { Prediction of bleeding events associated with warfarin } \\
\text { (dose selection) }\end{array}$ & [26] \\
\hline $\begin{array}{l}\text { Serum PSA level ( } 4 \mathrm{ng} / \mathrm{ml} \text { as the } \\
\text { upper limit of normal) }\end{array}$ & $21 \%$ & $94 \%$ & Prostate cancer: diagnosis and monitoring & [27] \\
\hline Serum AFP 30ng/ml & $65 \%$ & $89 \%$ & Liver cancer: diagnosis & [28] \\
\hline Serum AFP + VEGF + AFU & $100 . \%$ & $95 \%$ & Liver cancer: diagnosis & [29] \\
\hline HER2-FISH & $96-98 \%$ & $100 \%$ & Breast cancer: patient selction: use of trastuzumab & {$[30,31]$} \\
\hline HER2-IHC (Hercep) $2+, 3+$ & $66 \%$ & $98 \%$ & Breast cancer: patient selection: use of trastuzumab & [32] \\
\hline
\end{tabular}

The sensitivity and specifcity values in the table represent the association of the biomarker to relevant clinical events and may vary depending on factors such as clinical phenotype and cutoff values.

AFP: $\alpha$-fetoprotein; AFU: $\alpha$-fucosidase; FISH: Fluorescence in situ hybridization; HER2: Human epidermal growth factor receptor-2;

IHC: Immunohistochemistry; PSA: Prostate-specific antigen; UGT: UDP-glucuronosyltransferase: VEGF: Vascular EGF.

\subsection{Classification of Biomarkers}

One of the aims of personalising medicine is to use the growing advancements in the field of biology so that patients receive the appropriate drug for their disease, the right doseat the right time. Although the definitions of personalising vary, they all incorporate the use of different biomarkers driven by a decision-making process in which a diagnostic test is cardinal. Biomarkers include gene expression products, metabolites, polysaccharides, circulating tumor cells, peptides, proteins and other molecules such as circulating nucleic acids in plasma and serum. Ideal biomarkers used in early detection, diagnostics and prognostics, and for drug development and targeted therapies, mustbe highly specific and sensitive [5].

- Biomarkers can be categorised as Pharmacodynamics, prognostic or predictiveon the basis of their decision making capability in early drug development[6]:

1. Pharmacodynamics biomarkers: These biomarkers are molecular indicators which signal the outcome of the drug regimen and target interaction, including both therapeutic and inimical effects [7]. These biomarkers are especially used for dose optimization studies. These biomarkers are used in preclinical and phase I of clinical studies.

2. Prognostic biomarkers: These biomarkers indicate the likely course or progression of a disease in an untreated individual.[8] These biomarkers provide reliable progression of a disease and its consequent outcome irrespective of the treatment $[9,10]$. These biomarkers are used in phase II and III. Examples include PSA level at the time of diagnosis of prostate cancer, PIK3CA mutation status of tumor in women and many more.

3. Predictive biomarkers: These are disease- related biomarkers. It predicts how an individual will respond to the specific proposed treatment $[8,9]$. These markers are used in clinical phase II and III where the efficacy of the drugs to therapeutic intervention is monitored (also called as pre marketing trial) [10].

- Biomarkers can also be classified into two types on the basis of another parameter: Drug related and Disease related. 
Drug related biomarker detects the effect of specific drug on an individual suffering with the disease and how the patient's body is going to process it, assessing the therapeutic and adverse effects of the drug. However, the disease related biomarkers predicts the response of a particular treatment to a patient (Predictive Biomarker) and also tell about the most likely course of the disease regardless of the treatment given to an individual (Prognostic Biomarker).

\section{Clinical Biomarkers}

The clinical biomarkers determine the characteristics of a biomarker so that it is at par with an ideal biomarker and hence can be validated to for it diagnostic use in the markets. The transfer of the discovery of the biomarker to its validation for clinical use is active but is an emerging field of endeavour. As there are various limitations and pitfalls in translation of biomarker discovery to it clinical utility, the clinical biomarkers have been classified into various categories. These are basically the parameters on which the biomarkers are tested and validated to be approved as the clinical biomarkers. The figure [1] showed below gives us the brief overview of various parameters of a clinical biomarker.

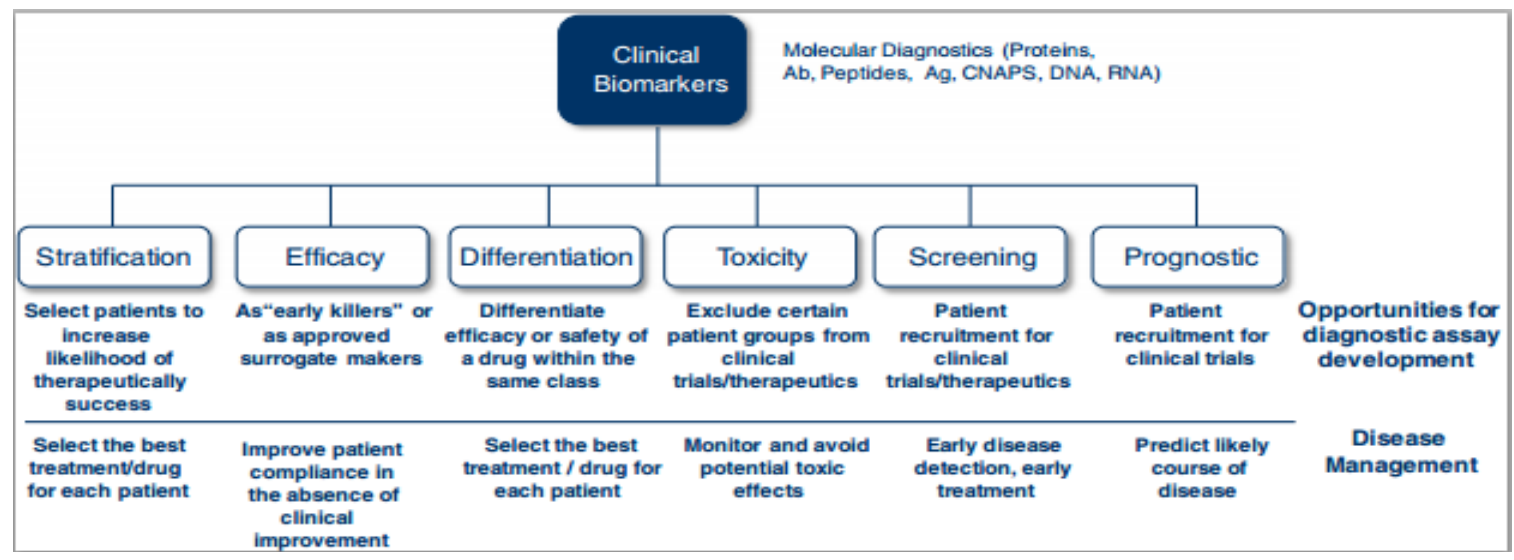

Fig1. Clinical biomarkers: categories/types [3]

- Figure 1, shows the resourcefulness of the clinical biomarkers. It determines a set of patients who share same biological characteristics to identify the right treatment for the patient(by stratification markers), decides the most effective and appropriate dose for the patient (by efficacy markers), determines the most specific therapy among the wide range of therapy availability for the patient which has negligible side effect (differentiation), determine the underlying vulnerability of a patient for a particular side effect or group of side effects due to the drug or the therapy (by toxicity markers), useful for early detection of the disease by assessing the symptoms(Screening markers) or assess the course and effectiveness end point of a therapy (by surrogate endpoint markers).

- Biomarkers as surrogate end points: These markers substitute the clinical biomarkers and correlate the effect of a specific treatment to the clinical outcome (like how a patient feels, responds to the drugs, functions, or how many patients survive) [9, 11, and 12].

The Biomarkers and Surrogate End Point Working Group [13] has classified the biomarkers on the basis of genetic and molecular biology [14]:

1. Type 0-Natural history: These are measures of natural history of a disease that correlates with known clinical outcomes.

2. Type I - Drug activity: It tracks the effects of therapeutic intervention associated with drug mechanism of action.

3. Type II- Surrogate endpoints: It consists of surrogate end points that is used as a complement of clinical biomarkers and predicts clinical benefit based on Patho physiological or other scientific evidence.

\section{Companion Diagnostics}

- Measurement of different markers (RNA, DNA, peptides and/or proteins) needs different qualification and validation strategies. Pharmaceutical companies are progressively looking for the 
development of a drug and diagnostic test simultaneously. The process where personalized medicine and drug-diagnostic go hand in hand is referred as companion diagnostic (CDx). The companion diagnostic is a diagnostic test which provides information about the applicability and efficacy of the specific drugor biological product, to better determine the appropriate patient population for treatment [3].Much emphasis is given on CDx asthey have proved to be an important tools in drug development due to the following results:

1. Cost reduction through stratification of patient population.

2. Higher chances of approval

3. Reduce time for analysis

4. Significantly increased market uptake and hence, added value for core business

- The journey of companion diagnostic started with the introduction of the drug - Herceptin (Trastuzumab; Roche/Genentech, 1998) for the detection of early stages of breast cancer and has now been in the market for more than a decade. Regulatory hurdles have been mentioned as one of the main reasons for the passive growth in this area. The discrepancies between the regulatory process in the European Union (EU) and USA and the convolution of the regulatory processes in both regions creates huge problems for companies. These difficulties affect the documentation and are more time consuming. However, the number of drugs marketed alongside CDx remains small (see Table 2).

Table2. Overview of already approved CDX on the markets [3,5]

\begin{tabular}{|l|l|l|l|}
\hline Biomarker & Drug & Year of approval & Indication \\
\hline HER-2/neu & Herceptin & 1998 & Breast cancer \\
\hline CD33 & Mylotarg & 2000 & Acute Myeloid Leukamia \\
\hline Estrogen Receptor & Faslodex & 2002 & Breast cancer \\
\hline CD20 & Bexxar & 2003 & Lymphoma \\
\hline EFGR & Erbitux & 2004 & Colorectal Cancer \\
\hline BCR-ABL Translocation & Dasatinib & 2005 & CML \\
\hline KRAS Mutation & Cetuximab & 2006 & Colorectal cancer \\
\hline HER2 Amplification & Trastuzumab & 2008 & Breast Cancer \\
\hline ALK Fusion & Crizotinib & 2011 & NSCLC \\
\hline $\begin{array}{l}\text { c- kit protein/ CD 117 antigen } \\
\text { expression }\end{array}$ & Imatinib Mesylate & 2012 & GIST \\
\hline EFGR Mutation & & & Melanoma \\
\hline BRCA1/2 gene defect & Tramatenib & 2013 & Ovarian Cancer \\
\hline
\end{tabular}

- These CDx have proved to be valuable and due to its remarkable results in the clinical assessments of tumor identification at the early stages, the demand for CDx has been increased greatly.

- The transition from discovery of biomarker to its validation is a complex procedure. Three aspects of measurements are taken into consideration during validation [59].1. Analytical or content validation, which ensures the consistency of the test in and showcase the degree to which the biomarker respond to the biological phenomenon. 2. Construct validity, which identifies the other relevant characteristics of the disease. 3. Criterion validity, which concerns with the sensitivity and specificity of the biomarker to a disease.

Table3. Strategic considerations and implications of personalised medicine [3]

\begin{tabular}{|l|l|}
\hline Pharmaceutical companies & $\begin{array}{l}\text { Generate new revenue system } \\
\text { Increased targeted therapies } \\
\text { Reduced clinical trials } \\
\text { Advancement in current clinical trials design }\end{array}$ \\
\hline Diagnostic companies & $\begin{array}{l}\text { Need to establish relationships with pharmaceutical companies } \\
\text { for cost effective and less time consuming process }\end{array}$ \\
\hline Payers/ Health ensurers & $\begin{array}{l}\text { Agree on reimbursements } \\
\text { Could alleviate the escalating healthcare costs }\end{array}$ \\
\hline Regulatory authorities & Reliable statistical relevance of clinical trials \\
\hline
\end{tabular}




\section{VALIDATION OF BIOMARKERS}

The transition of biomarkers from its discovery to the clinically tested diagnostic is a complicated and a rigorous procedure. The biomarker or we can say assays are processed through various parameters to authenticate itself for clinical use and determine the risk factors of the disease with extreme precision, specificity and sensitivity. The process can be broadly categorised into two groups:

1. Laboratory Validation: It is the characterization of a genetic marker. It evaluates the crucial characteristics: dose response, variability, reliability, sensitivity to a given procedure. This procedure has three main sources of error: a. Error in laboratory equipment's, b. Error due to biological change in an individual, c. Error due to alterations in biological products from time to time in a person $[61,75]$.

2. Population Validation: It is the validation of the biomarker for its usage on large no. of patients to evaluate the risk factors, determine the optimal handling and comprehending interpersonal variability. The crucial part of population validation is that the exposure assessment must be considered equally as the marker characteristics [61].

\section{BENEFITS OF BIOSENSOR THERAPY}

- Improved cardiovascular performance

- Stronger heart beats

- Stronger Adrenal Glands

\section{Advantages over other measurement scheme}

- They can measure non polar molecules that do not respond to most measurement devices.

- Disease mechanism are studied thoroughly before implementation

- They allow rapid continuous control.

- Highly specific and sensitive.

\section{Disadvantages}

- Heat sterilization is not possible as this would debase the biological part of biosensor.

- The membrane separating the reactor media from the immobilized sensor cell can become fouled by deposits.

- The cell in the biomarkers can become intoxicated by the other molecules that are capable of diffusing the other membranes.

- Changes in the reactor Ph can put chemical and mechanical stress on the biosensor that eventually impair it.

\section{BENEFITS OF BIOMARKERS}

The potential benefits of the biomarker stretch across the drugs life-cycle from helping to accelerate pre-clinical and clinical drugs development to assisting in post approval safety surveillance. For these reasons clinicians, regulators and insurers increasingly embraces biomarkers as it enables safer, more effective and less costly therapy.

\section{Discussions And Results}

\subsection{Cancer Detection and Diagnostics}

\subsubsection{Cancer Classification}

Cancers are illustrated by their pathological grade and clinical stage. Cancer develops steadily from changes in cells' genetics structure. Cancer is classified on the basis of site of origin of the malignant cell, cell structure analysis (grading) and expansion of the disease; the clinical stage of tumors from small localized cancers to metastatic cancer (staging). Integrated model of cancer classification is 
used for cancer identification and treatment [62].This model considers each attributes of the cancer and hence enhance the ability of the biomarker to target the components of the cancer distinctly for maximum therapeutic effect. The cancer stem cell model explains origin of cancer malignant cells, its initiation, progression and metastasis [63,64,65]. These stem cells have their distinct microenvironments to allow their specific functions. Advanced high through-put molecular analysis technologies, have simplified the molecular explanation of tumors. This molecular information is incorporate into the conventional classification system to improve cancer classification and has proved to be of potential use to biomarkers. As the cancer cells are blessed with capability for uninhibited propagation, invasion and metastasis, the integration model is the best way to classify the cancers effectively.

\subsubsection{Cancer Detection and Diagnostics}

Earlier cancer detection and accurate diagnosis is beneficial and important to improve the treatment chances and its outcome for cancer patients. Presently, the most valuable cancer diagnostic and prognostic indicators are morphological and histological traits of tumors or single biomarkers [2], such as prostate specific antigen (PSA). However, use of molecular tools is being augmented, both genomic and proteomic, to study molecular alterations in tumors and generatesmolecular signatures which sheds valuable insight in detection of the disease. Since tumor advancement includes many biological changes, these molecular signatures can be very complex. Correlating the molecular signatures with clinical parameters aids to provide stratified information that can be used by physicians to make decisions such as selection of effective cancer treatment. The major challenge to the research and development community is to evaluate these complex molecular signatures in the clinical setting.

\subsection{Cancer Biomarkers}

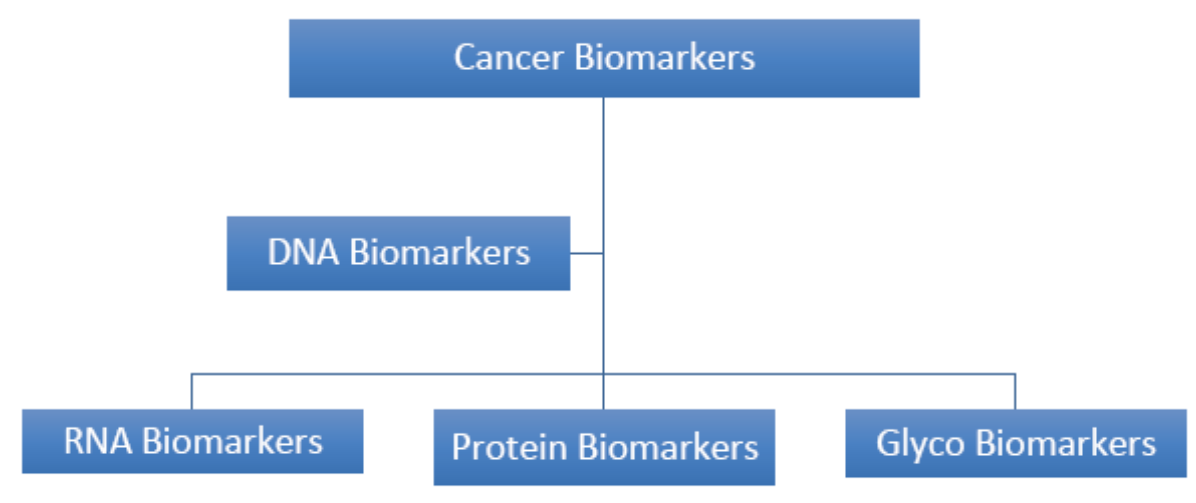

Cancer biomarkers are molecular alterations that can be detected in the tumor or in the blood, urine, or other fluids present in the body of cancer patients. These biomarkers or molecular signatures can be produced either by the tumor itself or by the body in response to the presence of cancer. These molecular signatures are assayed in collected bio-fluids like blood or serum non-invasively. Genetic, Glycomic, proteomic, DNA, RNA or protein modifications or other biological molecules are used for cancer diagnosis and prognosis. Cancer biomarkers can be used in several ways (NCI, 2005):

Risk, screening and detection markers: These markers are used for early detection of cancer symptoms in the patients. They are used to screen asymptomatic individuals who are at risk of cancer such as testing for mutations in BRCA1.

Diagnostic markers: These markers are used for identification, characterization and monitoring progression of cancer. These markers can be used at any stage of the cancer development [66]. They are also used to diagnose diseased individuals among those presenting with equivocal symptoms, or to differentiate among subclasses of disease. Some of the important biomarkers include PSA for detection of recurrence of prostate cancer, CA-125 monitoring for recurrence of ovarian cancer or HPV for uterine and cervical cancer. 
Prognostic markers: These markers indicate the possible outcomes of the malignant tumors in the untreated individual (tumorrecurrence, patient survival) which helps the clinicians to determine the most appropriate therapy .These markers are useful for the prediction of relapse of oral cancer. For example, in many solid tumors the spread of cancer cells to lymph nodes indicates an increase probability of tumor recurrence.

Predictive markers: These markersindicate the effectiveness of response of a drug to a specific therapy in the patient. These markers aid the doctors to select appropriate chemotherapeutic agents for the patient to achieve minimal side effects. For example, breast cancers that intends the estrogens receptor tend to respond to hormonal therapies such as tamoxifen [4], Gleevec targets certain subsets of Leukamia which has Philadephia chromosome[67].

The key elements in the evaluation of biomarkers are the analytical sensitivity and specificity of the assay to detect biomarkers in the context of the intended clinical use. Although many studies have looked for definitive coalition between clinical endpoints and cancer biomarkers, there is still a shortage of clinically meaningful markers that can benefit oncologists make patient management decisions. There are many possible explanations for the lack of markers which includes variable patient populations, tumor characteristics, treatments, and assay methods in the studies to identify, characterize and validate potential cancer biomarkers [15]. The National Cancer Institute (NCI) is placing significant emphasis on research to corroborate and validate biomarkers for clinical application.

\subsubsection{Dna Biomarkers}

The DNA biomarkers have proved to be a significant discovery in the domain of cancer. The sources of DNA are from tissue, sputum, saliva, serum, cerebrospinal fluid, bronchial tear and tumor cells. The best part of DNA markers is that they are found circulating in the blood, bone marrow, and nipple aspirate $[68,69,70]$. Besides nuclear aberrations, biological changes in mitochondrial DNA (mtDNA) molecules are recommended strongly as biomarkers for numerous cancers [68, 71, 72]. Apart from its potential of detection and prognostic method, it is also useful for monitoring tumors progression and drug effectiveness on the patient. These markers are used at every stage of cancer to predict and monitor the drug effectiveness and tumor progression. Single nucleotide polymorphisms (SNP) in most of the genes are major DNA markers. ATM, XRCC1, p53 (head, lung and neck cancers); CYP1A1, RAD1, BRCA1 and BRCA2 (breast cancer); and PGS2 (lung cancer) are some other DNA markers indicating different cancers. DNA markers involves loss of heterozygosity, chromosomal eccentricity at a gross cytogenetic level, such as translocation/fusion (BCR-ABL, PML-RARA translocation in leukaemias), epigenetic modifications and micro-satellite instability (MSI) [69, 66, 70, and 74]. These markers have proved to be useful due to its non-invasive nature, less risk factors then biopsies and moreover it is found in blood [73].

\subsubsection{Mirna Biomarkers}

Mirna are tiny non-coding RNA's and it acts as master regulators in various cellular processes. These markers can be detected in blood, serum, plasma and due to its non-evasive nature it is being used in early detection of cancers. It is been used for detection of pancreatic cancers. Cell free miRNA are found within apoptotic bodies, exosomes and complexed with AGO proteins, which prevents them by the action of RNA's and hence facilitating their isolation from normal cells. miRna acts as a tumor suppressor and oncogene. For example, miR15a is a suppressor for Bcl-2 in prostate cancer, chronic lymphocytic leukemia (CLL), and myeloma. let-7 is a suppressor for RAS in gastric and lung cancers and are oncogenic for many lymphomas; blastomas. Methods to detect miRNA include Serial analysis of Gene Expression (SAGE), micro analysis,differential display, and Quantitative Reverse Transcription Polymerase Chain Reaction (RT-qPCR). It is helpful in correlating disease diagnostics and therapeutic outcomes. 


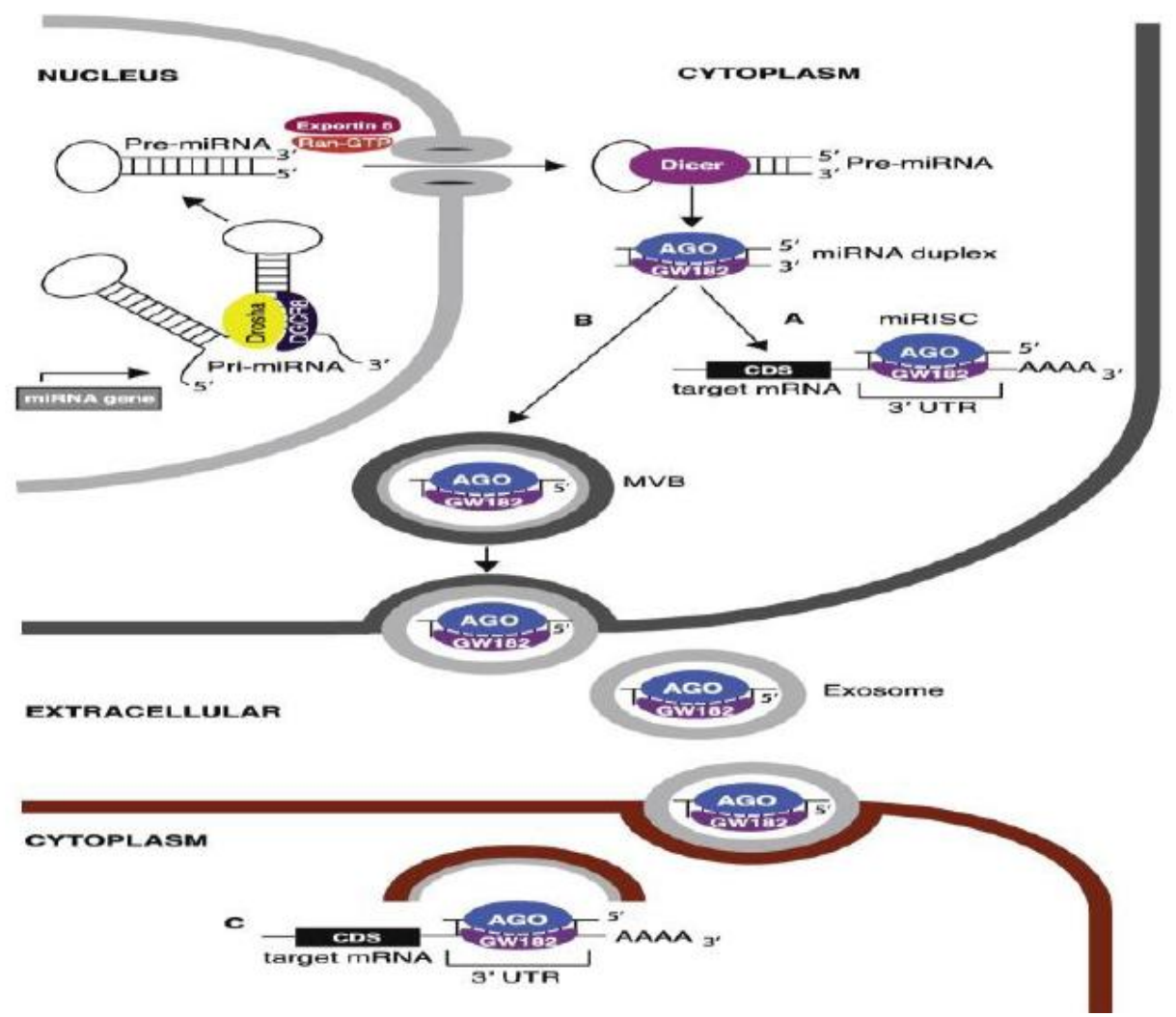

Figure3. A) Intracellular miRNA processing and function. B) Extracellular release of mature miRNA. C) Modulation of transcription by exogenous miRNA [74]

\subsubsection{Protein Biomarkers}

Proteins are the main killer bio-molecules in cells and that makes protein-based markers more important biomarkers than DNA- or RNA-based markers. Proteins controls the pathways of normal and transformed cells and this made them more relevant for disease initiation and progression. Examples of tumor markers include: CA 15-3 and prostate-specific antigen (PSA), for breast cancer and CA 125 for ovarian cancer. These markers have proteins whose expression is evaluated by immune his to chemical assays in fixed sections of tumor tissue. Moreover, the enzymatic activity of proteins can be used as a marker. Proteomics approaches have been used to produce a protein expression map (PEM) of the normal human breast, which is compared to the PEMs of breast cancer cells. Quantum Dots and nanotechnology are being recently used for the evaluating the potential of biomarkers.

\subsection{Microarrays and Cancer Analysis}

DNA microarrays are analytical tools consisting of a myriad of unique DNA sequences attached to a solid support. Microarrays can be used to examine DNA or RNA sequences derived from tumor cells. Microarrays can examine the expression of almost all the genes in the genome simultaneously. Research groups have begun to distinguish subsets of genes that show differential, coordinated expression between healthy tissues and their tumor counterparts. These molecular signatures are potential biomarkers for several types of cancer, including oral, breast, ovarian, lung, oral, colorectal and prostate cancers as well as lymphomas, melanomas, and leukaemia's [20]. Some biomarkers are useful in classifying tumors and determine response to therapies. Moreover, microarray data on differential expression can yield information about the role of both individual genes and pathways in cancer. Besides gene expression, microarrays are also used to genotype single nucleotide polymorphisms (SNPs) in oncogenesor tumor suppressors, analyze the relative copy number of genes to detect imbalances such as gene amplification [21] and execute genome-wide detection of LOH [22].

\subsection{Genomic and Proteomic Information for Biosensor-Based Cancer Testing}

Proteomic and genomic analyses produce a large volume of data but these approaches are primarily research tools that may not be pragmatic for regular cancer clinical testing because of their complexity. The tedious and lengthy protocols create issues of reproducibility and must be performed 
by highly skilled technicians. However, many of the complex patterns produced by genomic and proteomic analyses can be displayed by a smaller, more manageable number of biomarkers. For example, an assay that appraise the likelihood of breast cancer recurrence in women was developed using only 16 target genes analyzed performing a reverse transcriptase polymerase chain reaction (RT-PCR) assay [23]. This limited set of targeted genes was selected after analysis of the expression of 250 candidate genes selected from diversified sources. The assay was able to determine risk of disease recurrence and to predict patients who will benefit from chemotherapy for cases with nodenegative, estrogens-receptor positive breast cancer. Once a gene expression of complex pattern is reduced to a few genes, biosensor-based detection becomes more pragmatic and advantageous for cancer clinical testing, since it is faster, user friendly, less expensive and less technically demanding than microarray or proteomic analyses. However, more progress and research is still needed technically to improve the performance of protein based biosensors.

\subsection{Ligands for Cancer Clinical Testing}

Biosensors have been developed for protein analysis, depicting their potential for detecting protein biomarkers of cancer. However, one of the major barriers for multi-target cancer testing is the availability of recognition ligands. While antibodies are excellent ligands in terms of selectivity and binding strength, they are difficult to produce, incompatible with some high-throughput approaches, and generally have restrained shelf life. The crucial role of antibodies as the recognition ligand in biosensor systems makes this bottleneck demanding. There has been considerable interest in research on alternatives to antibodies. Several very promising avenues of investigation have been pursued including, protein-binding nucleic acids which bind other molecules with high affinity, [24,25,26] (KD 10-9 M), aptamers , peptides selected from phage display libraries [27,28], combinatorial chemistry ligands, scaffolded peptides and combinatorial chemistry ligands derived from low-affinity ligands. The ligands equilibrium dissociation constant $(K D)$ for antibodies of high affinity, is on the order of 10-9 M whereas, ligands that are peptides, scaffolded peptides, combinatorial chemistry ligands typically have $K \mathrm{D}$ 's of the order approximately $\sim 10-6$ to $10-7 \mathrm{M}$. A new generation of high affinity and versatile ligands compatible with high throughput systems is a key element for biosensor development and utilization.

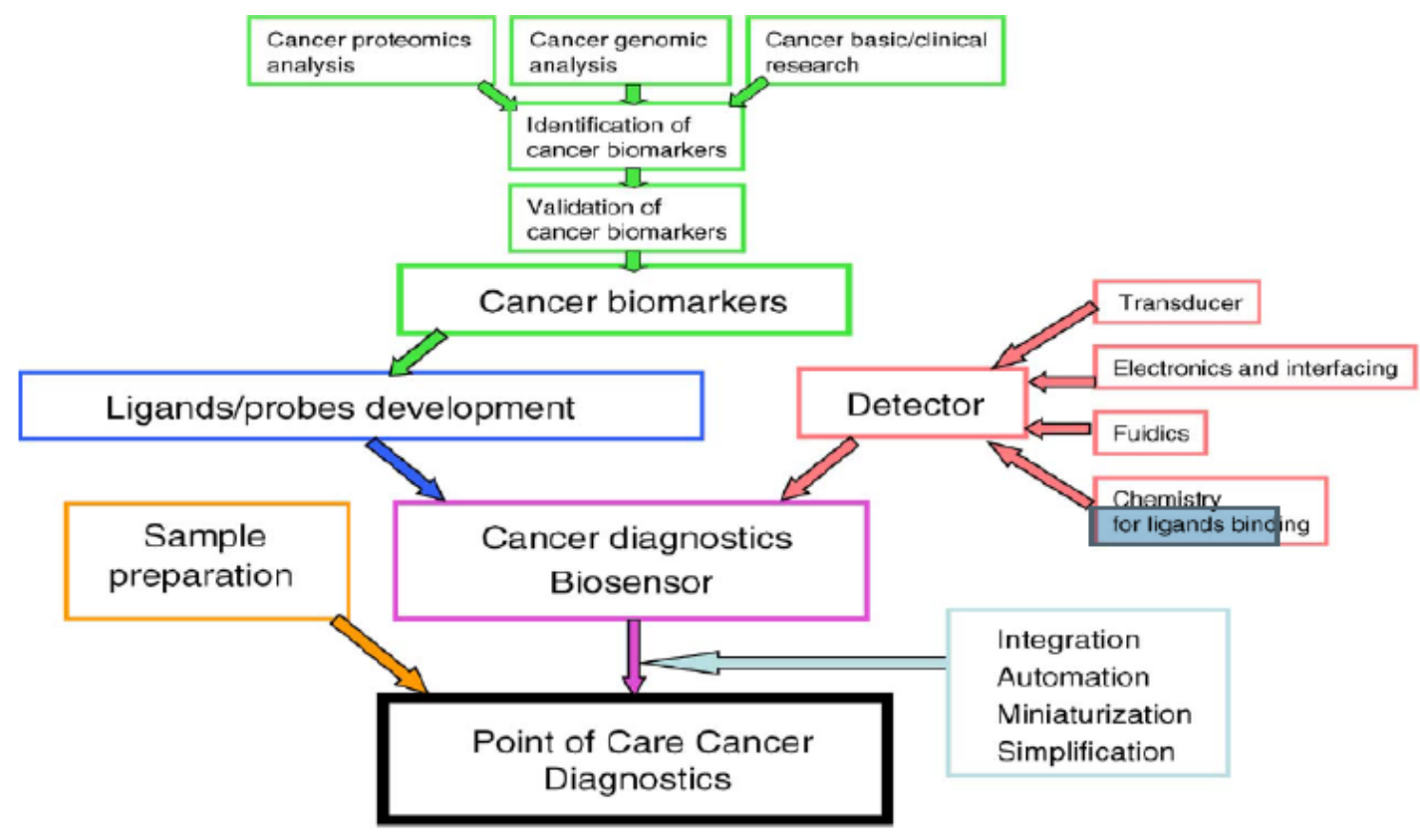

Figure4. The main elements required to adapt biosensors for cancer clinical testing. Cancer biomarkers identified from basic and clinical research, and from genomic and proteomic analyses must be validated. Ligands and probes for these markers can then be combined with detectors to produce biosensors for cancerrelated clinical testing. Point of Care cancer testing requires integration and automation of the technology as well as development of appropriate sample preparation methods [56].

\subsection{Sample Preparation and Cancer Cell Enrichment}

Sample preparation exhibits another major hindrance to the extensive use of biosensors in clinical applications. Conventionally, cancer diagnosis is carried out utilizing paraffin-embedded tumor tissue 
and/or analysis of individual biomarkers in blood. However, paraffin is not the optimal preservation method for the DNA, RNA and protein in various biosensor-based analyses. Nucleic acids retrieved from paraffin-embedded tissues are far more degraded than those recovered from fresh tissues even after successful PCR [29, 30, 31, 32, 33, 34]. Similarly, mRNA has been successfully segregated from archival tissue and was found accurate in some cases. [30, 35, 36, 37, 38, 39, 32, 24]. Proteins are even more difficult to recover from paraffin-embedded samples than nucleic acids [40]. Protein analysis is one of the major applications for biosensors, and the fact that proteins are not susceptible to analysis following rational fixation methods signals that fresh samples must be analyzed. For pragmatic outcomes, biosensor-based technologies for cancer clinical testing must incorporate welldefined sample collection procedures and enrichment systems to conversely recover different biomarkers from tumor cells and it becomes necessary to separate few targeted cancer cells from a large volume of normal cells. Various enrichment methods to select targeted cancer cells have been developed which includes density gradient centrifugation [41,42] providing mean tumor cell recovery rates of $\sim 86 \%$, counter-flow centrifugal elutriation (CCE) [43], immuno magnetic enrichment of cancer cells (more specific) [44,45,46,47,48 49,50] that allows 2300-fold [51] or 8139-fold enrichment. Flow cytometry is a process that separates cancer cells using several biomarkers simultaneously [51, 52, 53, 54]. Some enrichment system separates tumor cells based on their mechanical characteristics using a massive parallel micro fabricated sieving device $[55,76]$.

\subsection{Challenges in Moving Biosensors to Point of Care testing}

The ultimate prospect for biosensor application is Point of Care Testing (POCT). Point of care applications has great potential for cancer clinical testing. There are some challenges in transforming biosensors to POCT for cancer like:

- Improvement of reproducible biomarker assays.

- Advancement in recognition ligands.

- Amelioration of multi-channel biosensors.

- Miniaturization, simplification, automation and integration.

- Development of more sensitive, specific and robust transducers.

- advanced manufacturing techniques.

- cost reduction.

\section{Challenges and potential Solution}

The number of biomarkers in clinical settings is very less because of the following reasons:

(i) Sample cell preparation, enrichment and storage is not uniform

(ii) Analytical validation of equipments used is not adept.

(iii) Some techniques, such as mass spectrometry, provide large volume of data which has not been analyzed efficiently

(iv) Heterogeneity of cancer samples and most of the samples have not been collected and analyzed by laser capture microdissection

(v) Proper validated biomarkers are very less in number, due to its high precision requirement.

Large efforts have been taken by the FDA for the validation of biomarkers. In methylation field real time quantitative PCR and direct sequencing has been standardised. Efforts are also been made to do methylation profiling, miRNA profiling and his tone modifications in the same sample to reduce variation. Multi biomarker analysis is encouraged for minimal variation. Measurements of tumor markers and biopsy results are combined together for accurate results.

\section{CONClusion}

Lung cancer has the highest mortality rate among all cancer typesdue to the short survival time and quick invasion of the malignanttumours. While in women breast cancer is the main reason for morbidity due to its heterogeneous complexity. There may be some challenges and limitations to the use biomarkers but they offered great potential for monitoring and managing cancer at every stage 
ranging from screening, detection, diagnosis, prognosis to the treatment selection and assessment of therapies. With the advent of technology, the demand of companion diagnostics, transformation of biosensors to point of care testing is increasing due to its high speed, multi target analyses and great efficacy.

\section{ACKNOWLEDGEMENTS}

We have taken efforts in this research work. However, it would not have been possible without the kind support and help of many individuals and organizations. We would like to extend our sincere thanks to all of them. We would like to express our gratitude towards Hon. Chancellor SVKM's NMIMS; Management; Higher Authorities and Faculty Members for their kind co-operation and encouragement which helped us a lot. We would like to express our sincere thanks to industry persons and fellow colleagues for their kind attention and time.

\section{REFERENCES}

[1] Robert J. Torphy, Christopher J. Tignanelli, Joyce W. Kamande, Richard A. Moffitt, Silvia G. Herrera Loeza, Steven A. Soper, Jen Jen Yeh, Circulating Tumor Cells as a Biomarker of Response to Treatment in Patient-Derived Xenograft Mouse Models of Pancreatic Adenocarcinoma, PLOS ONE, February 2014 Volume 9 Issue.

[2] Shashikant Patil, Chinmaya Vyas, Biosensors: A Comprehensive Review and Its Role in Cancer Treatment, International Journal of Innovative Research in Electronics and Communications (IJIREC) Volume 3, Issue 3, 2016, PP 23-32 ISSN 2349-4042 (Print) \& ISSN 2349-4050 (Online) DOI: http://dx.doi.org/10.20431/2349-4050.0303004.

[3] Elisabeth Drucker and Kurt Krapfenbauer, Pitfalls and limitations in translation from biomarker discovery to clinical utility in predictive and personalised medicine, EPMA journal, DOI: $10.1186 / 1878-5085-4-7$

[4] Claudia Cava, Gloria Bertoli, Italo Zoppis, Giancarlo Mauri, Maria Carla Gilardi, Isabella Castiglioni, Candidate biomarkers for response to tamoxifen in breast cancer metastatic patients, 13th IEEE International Conference on BioInformatics and BioEngineering, vol. 00, no. , pp. 1-4, 2013, doi:10.1109/BIBE.2013.6701636

[5] Issaq HJ, Waybright TJ, Veenstra TD: Cancer biomarker discovery: opportunities and pitfalls in analytical methods. Electrophoresis. 2011, 32 (9): 967-975. 10.1002/elps.201000588

[6] Amur S, Frueh FW, Lesko LJ, Huang SM: Integration and use of biomarkers in drug development, regulation and clinical practice: a US regulatory perspective. Biomark Med. 2008, 2 (3): 305-311. 10.2217/17520363.2.3.305.

[7] Feuerstein GZ, Dormer C, Rufollo RR, Stiles G, Walsh FS, Rutkowski JL: Translational medicine perspectives of biomarkers in drug discovery and development. Part I. Target selection and validation - biomarkers take center stage. Int Drug Discovery. 2007, 2 (5): 36-43.

[8] Bruenner N: What is the difference between "predictive and prognostic biomarkers"? Can you give some examples? Connection. 2009, 13: 18

[9] Buyse M, Sargent DJ, Grothey A, Matheson A, de Gramont A: Biomarkers and surrogate end points - the challenge of statistical validation, National Rev. Clinical Oncology. 2007, 7 (6): 309317.

[10] Buyse M, Michiels S, Sargent DJ, Grothey A, Matheson A, de Gramont A: Integrating biomarkers in clinical trials. Expert Rev Mol Diagn. 2011, 11 (2): 171-182. 10.1586/erm.10.120

[11] Rosenkranz B: Biomarkers and surrogate end points in clinical drug development. Appl Clin Trials. 2003, 5 (2): 30-40

[12] Lassere MN, Johnson KR, Boers M: Definitions and validation criteria for biomarkers and surrogate end points: development and testing of a quantitative hierarchical level of evidence schema. J Rheumatol. 2007, 34 (3): 607-615.

[13] Atkinson AJ, Colburn WA, DeGruttola VG: Biomarkers and surrogate end points: preferred definitions and conceptual framework. Clin Pharmacol Ther. 2001, 69 (3): 89-95

[14] Frank R, Hargreaves R: Clinical biomarkers in drug discovery and development. Nat Rev Drug Discov. 2003, 2 (7): 566-580. 10.1038/nrd1130 
[15] Lisa M. McShane, Douglas G. Altman, Willi Sauerbrei, Sheila E. Taube, Massimo Gion, Gary M. Clark, Reporting recommendations for tumour marker prognostic studies (REMARK), European Journal of Cancer 41 (2005) 1690-1696, doi:10.1016/j.ejca.2005.03.032

[16] Dong G, Hutagalung AH, Fu C, Novick P, Reinisch KM,The structures of exocyst subunit Exo70p and the Exo84p C-terminal domains reveal a common motif. Nat Struct Mol Biol,Nature Structural \& Molecular Biology 12, 1094 - 1100 (2005), doi:10.1038/nsmb1017.

[17] Virginie Copois, Caroline Bret, Frédéric Bibeau, Jean-Paul Brouillet, Maguy del Rio, MarieLaurence Berthe, Thierry Maudelonde and Nathalie Boulle, Assessment of RNA Quality Extracted from Laser-Captured Tissues Using Miniaturized Capillary Electrophoresis, Lab Invest 2003, 83:599-602.

[18] Weibo Cai, Zachary J. Kerner, Hao Hong and Jiangtao Sun, Targeted Cancer Therapy with Tumor Necrosis Factor-Alpha, Biochemistry Insights 2008:1 5-21.

[19] Keith E. Herold, Avraham Rasooly, Biosensors and Molecular Technologies for Cancer Diagnostics,crc press, ISBN 9781439841655 - CAT\# K11937

[20] Macgregor $\mathrm{PF}^{1}$, Squire JA, Application of microarrays to the analysis of gene expression in cancer,Clin Chem. 2002 Aug; 48(8):1170-7.

[21] Xudong Huang,Craig S. Atwood,Robert D. Moir,Mariana A. HartshornRudolph E. Tanzi, Trace metal contamination initiates the apparent auto-aggregation, amyloidosis, and oligomerization of Alzheimer's $A \beta$ peptides,JBIC Journal of Biological Inorganic ChemistryDecember 2004, Volume 9, Issue 8, pp 954-960

[22] Catherine I Dumur, , Chavaboon Dechsukhum, , Joy L Ware, , Stacey S Cofield, , A.1 M Best, , David S Wilkinson, , Carleton T Garrett, , Andrea Ferreira-Gonzalez, Genome-wide detection of LOH in prostate cancer using human SNP microarray technology, Genomics, Volume 81, Issue 3, March 2003, Pages 260-269

[23] Soonmyung Paik, M.D., Steven Shak, M.D., Gong Tang, Ph.D., Chungyeul Kim, M.D., Joffre Baker, Ph.D., Maureen Cronin, Ph.D., Frederick L. Baehner, M.D., Michael G. Walker, Ph.D., Drew Watson, Ph.D., Taesung Park, Ph.D., William Hiller, H.T., Edwin R. Fisher, M.D., D. Lawrence Wickerham, M.D., John Bryant, Ph.D., and Norman Wolmark, M.D., A Multigene Assay to Predict Recurrence of Tamoxifen-Treated, Node-Negative Breast Cancer, N Engl J Med 2004; 351:2817-2826December 30, 2004, DOI: 10.1056/NEJMoa041588

[24] Michael Famulok, Andreas Jenne, Oligonucleotide libraries - variatio delectate, Current Opinion in Chemical Biology, Volume 2, Issue 3, June 1998, Pages 320-327, DOI: 10.1016/S13675931(98)80004-5

[25] Shaikh, K.A., Ryu, K.S., Goluch, E.D., Nam, J.M., Liu, J., Thaxton, C.S., Chiesl, T.N., Barron, A.E., Lu, Y., Mirkin, C.A., Liu, C., 2005. Proc. Natl. Acad. Sci. U.S.A. 102, 9745-9750.

[26] James R. Collett, E.J. Cho, J.F. Lee, Matthew Levy, A.J. Hood, C. Wan, , A.D. Ellington, Analytical Biochemistry 2005, Volume 338, Issue 1, pages 113-123

[27] Chang, C.Y., Abdo, J., Hartney, T., McDonnell, D.P., Mol. Endocrinol 2005

[28] Girja S. Shukla, David N. Krag, Phage display selection for cell-specific ligands: Development of a screening procedure suitable for small tumor specimens, Journal of Drug Targeting, Volume 13, 2005 -Issue 1, doi: 10.1080/10611860400020464

[29] Bielawski, K., A.Zaczek, U. Lisowska, A. Dybikowska, A.Kowalska, B.Falkiewicz,International Journal Molecular Medicine, 2001, Volume 8, 573-578.

[30] [A.Gloghini, B.Canal, U.Klein, L.Dal Maso, T.Perin, R.Dalla-Favera,A.Carbone, A., 2004. Journal of Molecular Diagnostics, Volume 6, 290-296

[31] S.J. Diaz-Cano, S.P. Brady, DNA Extraction from Formalin-fixed, Paraffin-embedded Tissues: Protein Digestion as a Limiting Step for Retrieval of High-quality DNA, Diagnostic Molecular Pathology, 1997, Volume 6, Issue 6, pages 342-346.

[32] Gregor Weirich, Maria Anna Hornauer, Thomas Bruning, H. Hofler, H. Brauch, Molecular Biotechnology,1997, volume 8, pg 299-301, DOI: 10.1007/BF02760783

[33] [33] Wang, H.Y., Luo, M., Tereshchenko, I.V., Frikker, D.M., Cui, X., Li, J.Y., Hu, G., Chu, Y., Azaro, M.A., Lin, Y., Shen, L., Yang, Q., Kambouris, M.E., Gao, R., Shih, W., Li, H., 2005. Genome Res. 15, 276-283. 
[34] J. Trojan, A. Brieger, J. Raedle, M. Esteller, S. Zeuzem, 5'-CpG island methylation of the LKB1/STK11promoter and allelic loss at chromosome 19p13.3 in sporadic colorectal cancer, 2000. Gut 47, 272-276, DOI:10.1136/gut.47.2.272

[35] Helene Nortvig Abrahamsen, Torben Steiniche, Ebba Nexo, Stephen J. Hamilton- Dutoit, Boe Sandahl Sorensen, Towards Quantitative mRNA Analysis in Paraffin-Embedded Tissues Using Real-Time Reverse Transcriptase-Polymerase Chain Reaction: A Methodological Study on Lymph Nodes from Melanoma Patients, The Journal of Molecular Diagnostics, Volume 5, Issue 1,Februray 2000, Pages 34-41, DOI: 10.1016/S1525-1578(10)60449-7

[36] Specht K., Richter T., Muller U., Walch A., Hofler M.W., Journal of Molecular Medicine, 2000, 78, B27

[37] Makoto Shibutani, Chikako Uneyama, Keiko Miyazaki, Kazuhiro Toyoda, Masao Hirose, Methacarn Fixation: A Novel Tool for Analysis of Gene Expressions in Paraffin-Embedded Tissue Specimens, Laboratory Investigation ,2000, 80: 199-208

[38] Susan M. Goldsworthy, Pat S. Stockton, Carol S. Trempus, Julie F. Foley, Robet R. Maronpot, Effects of fixation on RNA extraction and amplification from laser capture microdissected tissue, Molecular Carcinogenesis, Volume 25, Issue 2 , June 1999 ,Pages 86-91, DOI: 10.1002/(SICI)1098 2744(199906)25:2<86::AID-MC2>3.0.CO;2-4

[39] Giorgio Stanta, Serena Bonin, Rosella Perin, RNA Extraction from Formalin-Fixed and Paraffin-Embedded Tissues, Methods in Molecular Biology, 1998, volume 86,pg 23-26, ISBN: 978-1-59259-570-9(online)

[40] Kimimasa Ikeda, Takushi Monden, Toshiyuki Kanoh, Masaki Tsujie, Hikaru Izawa, Akinao Haba, Tadashi Ohnishi, Mitsugu Sekimoto, Naohiro Tomita,Hitoshi Shiozaki, Morito Monden, Extraction and Analysis of Diagnostically Useful Proteins from Formalin-fixed, Paraffinembedded Tissue Sections, Journal of Histochemistry. Cytochemistry,1998,vol 46, 397-403, doi: 10.1177/002215549804600314

[41] Valerie Choesmel,Jean-Yves Pierga, Claude Nos, Anne Vincent-Salomon,Brigitte Sigal-Zafrani, Jean- Paul Thiery, Nathalie Blin, Enrichment methods to detect bone marrow micrometastases in breast carcinoma patients: clinical relevance,2004, Breast Cancer Research, 6, R556-R570, DOI: $10.1186 / \mathrm{bcr} 898$

[42] Rosenberg, R., Gertler, R., Friederichs, J., Fuehrer, K., Dahm, M., Phelps, R., Thorban, S., Nekarda, H., Siewert, J.R., 2002. Cytometry 49, 150-158.

[43] Dlubek, D., Dybko, J., Wysoczanska, B., Laba, A., Klimczak, A., Kryczek, I., Konopka, L., Lange, A., 2002. Eur. J. Haematol. 68, 281-288.

[44] Veit Zieglschmid, Christiane Hollmann, Bertha Gutierrez,Winfired Albert, Dark Strothoff, Eberhard Gross, Oliver Bocher, Combination of Immunomagnetic Enrichment with Multiplex RT-PCR Analysis for the Detection of Disseminated Tumor Cells, Anticancer Research,2005,vol $25,1803-1810$.

[45] Ute Woelfle, Elisabeth Breit, Kristine Zafrakas,Marcus Otte, Falk Schubert, Volkmar Muller, J.R. Izbicki, Thomas Loning, Klaus Pantel, Bi-specific immunomagnetic enrichment of micrometastatic tumour cell clusters from bone marrow of cancer patients, Journal of Immunological Methods, 2005, vol 300, 136-145, DOI:10.1016/j.jim.2005.03.006

[46] Anja Ulmer, Oleg Schmidt-Kittler, Jorg Fischer, Ulf Ellwanger, Gernot Rassner, Gert Riethmuller, G. Fierlbeck, Christoph A. Klein, Immunomagnetic Enrichment, Genomic Characterization, and Prognostic Impact of Circulating Melanoma Cells, Clinical Cancer Research,2004,vol 10,pg 531-537,DOI: 10.1158/1078-0432.CCR-0424-03

[47] Oscar Lara, Xiaodong Tong, Maciej Zborowski, Jeffrey J. Chalmers, Enrichment of rare cancer cells through depletion of normal cells using density and flow-through, immunomagnetic cell separation, Experimental Hematology,2004,vol 32,Issue 10, pages 891-904, DOI: 10.1016/j. exphem.2004.07.007

[48] Richard E. Zigeuner, RainerRiesenberg, Heike Pohla, Alfons Hofstetter, Ralph Oberneder, Immunomagnetic Cell Enrichment Detects more Disseminated Cancer Cells than Immunocytochemistry in Vitro, The Journal of Urology, 2000, Volume 164, Issue 5, Pages $1834-1837$. 
[49] Martin R. Weihrauch, Edmund Skibowski, Andreas Draube, Andrea Geller, Hans Tesch, Volker Diehl, Heribert Bohlen, Immunomagnetic enrichment and detection of isolated tumor cells in bone marrow of patients with epithelial malignancies, Clinical \& Experimental Metastasis, 2002a, volume 19, 617-621, DOI: 10.1023/A:1020988227349

[50] Eric Kielhorn, Kevin Schofield C.T, DavidL. Rimm, Use of magnetic enrichment for detection of carcinoma cells in fluid specimens, Cancer, 2002, volume 94, pgs 205-211, DOI: $10.1002 / \mathrm{cncr} .10193$

[51] Hu X.C., Wang Y., Shi, D.R., Loo T.Y., Chow L.W.,Immunomagnetic Tumor Cell Enrichment Is Promising in detecting Circulating Breast Cancer Cells, Oncology, 2003,64, 160-165, DOI: $10.1159 / 000067776$

[52] Allison L.Allan, Sharon A. Vantyghem, Alan B. Tuck, Ann F. Chambers, Ian H. Chin-Yee, Michael Keeney, Detection and quantification of circulating tumor cells in mouse models of human breast cancer using immunomagnetic enrichment and multiparameter flow cytometry, Cytometry A,volume 65, 4-14,DOI: 10.1002/cyto.a.20132

[53] Petra S. Kraemer, Carissa A. Sanchez, Gary E. Goodman, James Jett, Peter S. Rabinovitch, Brian J. Reid, B.J, Cytometry A,2004, volume 60, 1-7, DOI: 10.1002/cyto.a.20041

[54] Markus Feuerer, Marian Rocha,Lianhua Bai, Victor Umansky, Erich-Franz Solomayer, Gunther Bastert, Ingo J.Diel, Volker Schirrmacher, Enrichment of memory T cells and other profound immunological changes in the bone marrow from untreated breast cancer patients, International Journal of Cancer, 2001, volume 92, Issue 1,Pages 96-105, DOI: 10.1002/10970215(200102)9999:9999<::AID-IJC1152>3.0.CO;2-Q

[55] H.Mohamed,L.D.McCurdy, D.H. Szarowski, S. Duva, J.N. Turner, M.Caggana, Development of a rare cell fractionation device: application for cancer detection, IEEE Transactions on Nanobioscience, 2004, Volume 3,Issue 4, Pages 251-256.

[56] Avraham Rasooly, James Jacobson, Development of biosensors for cancer clinical testing, Biosensors and Bioelectronics 21 (2006) 1851-1858

[57] Chinmaya vyas, Shashikant Patil, Amar khalore, comprehensive assessment of wearable smart textile sensors, International Journal of Advanced Information Science and Technology (IJAIST) ISSN: 2319:2682 Vol.45, No.45, January 2016.

[58] Shashi Amur, Felix W Frueh, Lawrence J Lesko, Shiew-Mei Huang, Integration and use of biomarker in drug development, regulation and clinical practice: a US regulatory perspective, Biomarkers medicine, 2008, Volume 2, Issue 3, pg 305-311

[59] Schulte PA, Perera FP. Validation. In: Molecular epidemiology: principles and practices (Schulte PA, Perera FP, eds), pp 79-107. San Diego: Academic Press, 1993

[60] Perera FP, Weinstein IB, Molecular epidemiology: recent advances and future directions, Carcinogenesis. 2000 Mar, Volume 21, Issue 3, Pg 517-524.

[61] Paul A. Shulte, D. Gayle DeBord, Public Health Assessment of Genetic Information in the Occupational Setting,Public health Genomics, 2000

[62] Idikio HA. Human Cancer Classification: A Systems Biology- Based Model Integrating Morphology, Cancer Stem Cells, Proteomics, and Genomics. J Cancer 2011; 2:107-115. doi:10.7150/jca.2.107

[63] M, Fuller M. Stem Cells and Cancer: Two Faces of Eve. Cell. 2006;124:1111-1115

[64] Jordan C, Guzman M, Noble M. Cancer Stem Cells. New Engl J Med. 2006;355:1253-1256

[65] Fodde R, The Stem of Cancer. Cancer Cell. 2009;15(2):87-90

[66] Verma, M.; Manne, U. Genetic and epigenetic biomarkers in cancer diagnosis and identifying high risk populations. Crit. Rev. Hematol. Oncol. 2006, 60, 9-18.

[67] Milone, J.H.; Enrico, A. Treatment of Philadelphia chromosome-positive acute lymphoblastic leukemia. Leuk. Lymphoma 2009, 50, 9-15.

[68] Verma, M.; Kumar, D. Application of mitochondrial genome information in cancer epidemiology. Clin. Chimica. Acta 2007, 383, 41-50.

[69] Sidransky, D. Emerging molecular markers of cancer. Nat. Reviews. Cancer. 2002, 2, 210-219.

[70] Leon, S.A.; Shapiro, B.; Sklaroff, D.M.; Yaros, M.J. Free DNA in the serum of cancer patients and the effect of therapy. Cancer Res. 1977, 37, 646-650. 
[71] Verma, M.; Srivastava, S. Epigenetics in Cancer: Implications for Early Detection and Prevention, Lancet Oncology, 2002, 3, 755-763.

[72] Verma, M.; Kagan, J.; Sidransky, S.; Srivasatava, S. Proteomic analysis of the cancer cell mitochondria. Nat. Rev. Cancer 2003, 3, 789-795

[73] Chatterjee, S.K.; Zetter, B.R. Cancer biomarkers: knowing the present and predicting the future. Future Oncol. 2005, 1, 37-50.

[74] Francesca Montani, Fabrizio Bianchi, Circulating Cancer Biomarkers: The Macro-revolution of the Micro-RNA, NCBI,https://dx.doi.org/10.1016\%2Fj.ebiom.2016.02.038

[75] Patil Shashikant, Sonawane Sachin, Upadhyay Nischay, Srivastava Aanchal, COMPRESSIVE ASSESSMENT OF BIOINFORMATICS IN BIOMEDICAL IMAGING AND IMAGE PROCESSING, International Journal of Computer Engineering \& Technology (IJCET) ISSN Print : 0976 - 6367; ISSN Online: 0976 - 6375.

[76] Suresh Kurumbanshi, Shashikant Patil, Performance analysis of monitored biomedical data using biotrace tool, International Journal of Emerging Technologies in Computational and Applied Sciences(IJETCAS) ISSN (Print): 2279-0047 ISSN (Online): 2279-0055

\section{Authors' BIOGRAPHY}

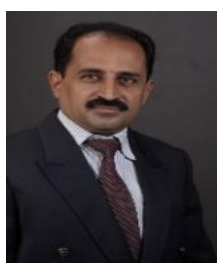

Prof. Shashikant Patil, is working as an Associate Professor in Electronics and Telecom Department in SVKM'S NMIMS, India. He is currently serving as an Associate Editor, Editorial Board Member, Technical Advisory Board Member, Potential Peer Reviewer and Journal Referee on at least 50 Journals. He is also having an association with Elsevier Editorial Series, Taylor and Francis, Springer Link Journals as a potential Peer Reviewer and Journal Referee. He is also associated with IEEE, ACM, CSI, ISTE, NHRDN, E4C and IETE as Professional member and nominated as an affiliate member on various committees of IEEE SPS and ComSoc. He is recipient of Best Researcher Award 2014 of SVKMs NMIMS Shirpur. He has published around 31 articles in various conferences and journals at National and International level. This year he has been selected as Regional Lead Ambassador for region 10 for IEEE Day 2015 and 2016 event. He is member IEEE RFID Technical Council and SIG Member of IoT. His research interests are Signal Processing and Imaging. He is also serving as an Editor in Chief for IEEE CRFID Newsletter.

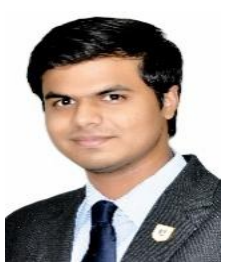

Chinmaya Vyas, is currently pursuing B. Tech in Electronics and Telecommunication Engineering from SVKM'S NMIMS, Mumbai, India. His research interest includes embedded system and robotics, antenna and radio frequency, sensors and control systems. He has published 06 articles in Conferences and International Journals.

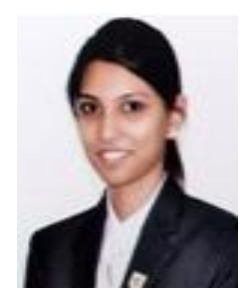

Bhumija Singh, is currently pursuing B. Tech in Electronics and Telecommunication Engineering from the SVKM'S NMIMS, Mumbai, India. Her research interest includes embedded system and robotics, microwave, biomedical, image processing. She has published articles in a national conference and an international conference. 